\title{
Competitive Neural Layer-based Method to Identify People with High Risk for Diabetic Foot
}

Ana Cláudia Barbosa Honório Ferreira ${ }^{1}$, Danton Diego Ferreira ${ }^{2}$, Henrique Ceretta Oliveira ${ }^{1}$, Igor Carvalho de Resende ${ }^{2}$, André Anjos ${ }^{3}$ and Maria Helena Baena de Moraes Lopes ${ }^{1}$

${ }^{1}$ School of Nursing, Universidade Estadual de Campinas, Campinas, São Paulo, Brazil

2 Automation Department, Universidade Federal de Lavras, Lavras, Minas Gerais, Brazil

${ }^{3}$ Idiap Research Institute, Martigny, Switzerland

*Corresponding author: Email: danton@ufla.br

\section{ABSTRACT}

Background and Objective: To automatically identify patients with diabetes mellitus (DM) who have high risk of developing diabetic foot, via an unsupervised machine learning technique. Methods: We collected a new database containing 54 known risk factors from 250 patients diagnosed with diabetes mellitus. The database also contained a separate validation cohort composed of 73 subjects, where the perceived risk was annotated by expert nurses. A competitive neuron layer-based method was used to automatically split training data into two risk groups. Results: We found that one of the groups was composed of patients with higher risk of developing diabetic foot. The dominant variables that described group membership via our method agreed with the findings from other studies, and indicated a greater risk for developing such a condition. Our method was validated on the available test data, reaching 71\% sensitivity, 100\% specificity, and 90\% accuracy. Conclusions: Unsupervised learning may be deployed to screen patients with diabetes mellitus, pointing out high-risk individuals who require priority follow-up in the prevention of diabetic foot with very high accuracy. The proposed method is automatic and does not require clinical examinations to perform risk assessment, being solely based on the information of a questionnaire answered by patients. 
Our study found that discriminant variables for predicting risk group membership are highly correlated with expert opinion.

Keywords: Diabetes Mellitus; Diabetic foot; Artificial Neural Network

\section{Introduction}

Diabetes mellitus (DM) is classified as a chronic disease, which occurs due to defects in insulin action in the body or as the result of problems in its secretion ${ }^{(1-2)}$. According to the International Diabetes Federation ${ }^{(3-4)}$, Brazil ranks fourth among the countries with the largest number of individuals with diabetes, with 12.5 million patients in 2017 , and a prevalence of 8 to 9\%. In 2017 alone, DM claimed 4 million lives in Brazil, with associated health spending totaling 29.3 billion Brazilian reais ${ }^{(5)}$ (approximately 7.15 billion US dollars). In Central and South America, there are about 32 million individuals with a positive diagnosis, and the mortality of DM is higher than the combined numbers of patients with HIV/AIDS, tuberculosis and malaria, with one death occurring every six seconds ${ }^{(3)}$. Finally, last year's statistics indicate that there are 463 million people from 20 to 79 years of age that have been diagnosed with DM worldwide, with a projected increase of $51 \%$ by 2045 .

There are various complications of DM, but this work focuses on risk assessment for the so-called "diabetic foot," which is one of the most serious and has high treatment costs, representing a major challenge for healthcare workers, resulting in significant morbidity ${ }^{(6-7)}$. Patients with DM and ulcers on the feet are more prone to develop depression and to experience a low quality of life. An early diagnosis and more resolute treatment at initial phases of this complication are necessary to control it more effectively ${ }^{(7-8,9)}$.

The percentage of amputations of limbs may be improved by $40 \%$ (from 0.8 to $0.5 \%$ ) when self-care actions by and methods of education for patients in relation to diabetic foot are 
implemented properly ${ }^{(10)}$. Proper care of the feet, good control of glucose, and education can prevent up to $85 \%$ of amputations of lower limbs in DM patients ${ }^{(8,11,12)}$.

Recently, the Brazilian government has pushed for improvements in primary healthcare $^{(13)}$ through specialized programs and multi-disciplinary research. In particular, the strategy manual for the care of people with $\mathrm{DM}^{(13)}$ promotes multidisciplinary follow-up specialized for individuals suffering from DM and has as its goals the education of diabetes patients and the prevention, early diagnosis, and early treatment of lesions in target organs, and, in particular, diabetic foot ${ }^{(13,14)}$.

The most effective way to prevent diabetic foot is early identification of patients at high risk of developing this complication ${ }^{(14,15)}$. To achieve this goal, we propose the use of an unsupervised learning technique to automatically classify the risk of developing diabetic foot. Our solution is based on a competitive neural network architecture, supported by patient data. The proposed method does not require clinical exams, allowing any healthcare professional to collect the necessary input variables (characteristics on the health conditions of patients and foot care) that are directly fed to an Artificial Neural Network (ANN) ${ }^{(16)}$. As the proposed system does not require clinical exams, the classification process is simple. The required information about the patient may be collected by an attendant, or by the patients themselves in a healthcare unit, and does not require the intervention of a specialized professional. In addition to risk classification, the questionnaire used for data collection provides valuable information about foot care, changes already noticed, and the patient knowledge about the disease. With this data, healthcare professionals can develop a plan of care specifically designed for the patient to prevent the development of diabetic foot. Given the prevalence of $\mathrm{DM}$, it is not possible to follow up all patients with frequent and detailed consultations ${ }^{(17,18)}$. The proposed system helps in the screening process, and it provides better care for patients with higher risk of developing diabetic foot. 
The remainder of this article is organized as follows. The next section presents a literature review about methods based on computational intelligence for diabetes and its complications. Next, we describe the multivariate dataset and its contents. We then formalize our unsupervised method for clustering patients into high- and low-risk groups and describe a univariate statistical analysis that was performed to identify the variables that most discriminate between high- and low-risk groups. Finally, we present our results and draw conclusions.

\section{Literature Review}

In our literature review, we explored the current methods for performing the assessment of diabetic foot, what risk factors have been used by the recent model-based methods to predict diabetic foot, and what artificial intelligence techniques are being used to perform diabetic foot prediction, screening, and identification. We also reviewed different approaches for diabetic foot ulcer (DFU) evaluation based on plantar pressure and infrared thermography.

Classical risk assessment methods are based on in-clinic evaluation through various tests including the Semmes-Weinstein monofilament examination for pain sensitivity, the tuning fork examination for perception vibration, palpitation of the dorsalis pedis and the posterior tibial pulses, and the identification of changes in the foot, such as claw toes, hallux valgus, and Charcot feet ${ }^{(17)}$. Such tests may be accompanied by a risk score recommended by the Wagner ${ }^{(19)}$ and the University of Texas Wound Classification Systems of Diabetic Foot Ulcers $^{(20)}$. The American Diabetes Association and the Scottish Intercollegiate Guideline Network have also been used for the classification of diabetic foot risk by performing tests to determine the loss of protective sensation ${ }^{(21,22)}$. These classical methods display a common need for clinical exams performed by healthcare professionals, especially nurses, to assess the patient's risk to develop diabetic foot ${ }^{(18)}$. It is worth mentioning that these clinical exams 
require experienced professionals and are often time-consuming to perform. Additionally, there is little information regarding the prediction capabilities of these methods in the available literature, as validation demands a large number of patients who developed or did not develop diabetic foot after a certain period of time ${ }^{(23)}$. As a result, the validation of systems of risk stratification for foot ulcers is perceived as challenging, and consequently, the identification of the most appropriate system remains undetermined ${ }^{(24)}$.

Unlike with the classical methods, easier to obtain clinical variables pertaining to patients have also been used to determine the level of risk ${ }^{(21)}$. Using such variables as input and statistical models derived from univariate and multivariate analysis have been proposed in the past ${ }^{(14,15)}$. The combination of risk factors is the key to improving the predictability of the data-based models. Most of the data-based models have in common the use of gender, age, duration of diabetes, prior amputation or ulceration, socio-economic and medical histories, and clinical and laboratory exams as input variables ${ }^{(25-27)}$.

With the aim of predicting diabetic foot, Farzi et al. (2017) ${ }^{(25)}$ focused on patients with type 2 diabetes. They experimented with various types of supervised classification architectures, and they presented a comparative analysis for each of the input variables considered in that study (patient's medical history, infection year, blood pressure, blood and urine test results, referral date, and treatment process, as well as observed complications). Random forest classifiers reached the highest accuracy $(95 \%)$. Heald et al. $(2018)^{(26)}$ combined risk factors glycated hemoglobin test (HbAlc), age, absence of monofilament sensation, creatinine level, and history of stroke) in a single logistic regression model achieving modest predictive power of diabetic foot, with an area under the receiver operating characteristic (ROC) curve of 0.65 . Crawford et al. $(2018)^{(27)}$ proposed a prognostic model of independent risk factors for foot ulceration in diabetes using all available individual patient data from cohort studies conducted worldwide. They conducted a systematic review and 
meta-analysis of individual patient data from 10 cohort studies of risk factors in the prediction of foot ulceration in diabetes. A logistic regression model was used to derive adjusted odds ratios (ORs) for foot ulceration subject to ulceration history, monofilament insensitivity, any absent pedal pulse, age, gender, and diabetes duration. Combining three predictors (foot ulceration history, monofilament insensitivity, and any absent pedal pulse) produced sensitivities from $90.0 \%$ to $95.3 \%$, with corresponding specificities from $12.1 \%$ to $63.9 \%$.

In relation to diabetic foot screening, detection, and prevention, most of the available literature concerning artificial intelligence (AI) techniques is devoted to two basic categories: i) risk assessment of diabetic foot after foot examination, or ii) screening of already existing DFUs, typically via image-based methods.

A variety of methods based on AI techniques have recently been applied to diabetic foot screening. The most common ones include $\mathrm{ANNs}^{(20,28,29,30,31)}$, fuzzy $\operatorname{logic}{ }^{(32,33)}$, and support vector machines $(\mathrm{SVMs})^{(34,35)}$. Most systems are trained using a supervised learning approach, where an input is mapped to an output based on example input-output pairs. Among ANN techniques used, the work reported by Signh et al. (2013) ${ }^{(29)}$ applied a multilayer perceptron (MLP) to evaluate the risk of DFU in patients with type $2 \mathrm{DM}$, whereas others have applied the convolutional neural network (CNN), which is more often recommended for image processing. Of the AI-based works, Wang et al. (2017) ${ }^{(34)}$, Goyal et al. $(2018)^{(28)}$, Goyal et al. (2019) $)^{(30)}$, Li et al. (2019) ${ }^{(33)}$, Wijesinghe et al. (2019) $)^{(20)}$, and Ohura et al. (2019) $)^{(31)}$ used images to analyze diabetic foot, while Gomes et al. (2015) ${ }^{(32)}$, Wang et al. $(2017)^{(34)}$, Goyal et al. $(2018)^{(28)}$, Goyal et al. (2019) $)^{(30)}$, Wijesinghe et al. $(2019)^{(20)}$, and Ohura et al. (2019) ${ }^{(31)}$ looked for alternatives to monitor already existing DFUs.

Different approaches based on plantar pressure include shoe designing ${ }^{(33)}$, and optical sensors $^{(34)}$ have also been developed for diabetic foot detection. Dynamic foot pressure measurement is necessary particularly for diabetic patients to prevent ulcers which can 
eventually lead to gangrene and amputation ${ }^{(34)}$. Plantar pressure off-loading at the ulcer site is one of the therapeutic interventions for healing DFU ${ }^{(36)}$. Due to peripheral neuropathy and vascular disease, the foot anatomy of patients with diabetes is significantly different from that of normal persons. The work reported in Priyadharshini et al. (2017) ${ }^{(36)}$ derived standardized foot anthropometric data for patients with DFU to use as a reference for developing pressure off-loading devices. Rabbani et al. (2013) ${ }^{(37)}$ developed a low-cost optical sensing system for dynamic foot pressure measurement for diabetic patients to prevent ulcers.

Another approach for the detection and prevention of DFU is the analysis of temperature distribution in the plantar region by means of infrared thermography ${ }^{(36)}$. Hernandez-Contreras et al. $(2016)^{(38)}$ presented a narrative review of diabetic foot and infrared thermography. They found that studies have demonstrated that temperature variations in the plantar region can be related to diabetic foot problems ${ }^{(38)}$. The core idea of the infrared thermography-based method is to submit the participant to external stress in the plantar region of the feet. Afterwards, the regions that present temperature differences before and after the stress are analyzed, which indicates an area at risk for injuries.

Our work presents an alternate route in preventive care for diabetic foot, with a solution for risk assessment that requires no previous clinical or laboratory examination and solely uses information provided by patients via a questionnaire. Therefore, the proposed method avoids both physical contact with the individual and foot imaging. The developed questionnaire collects information related to changes diabetic patients would feel in the feet, such as numbness and loss of sensation and/or tingling, as well as information about daily foot care such as the type of footwear commonly used. Such information is essential in building a sensitive system to predict the possibility of DM patients to develop diabetic foot, even before any visual changes can be perceived. 
Different from the methods designed using a supervised learning approach, the proposed method employs an unsupervised learning technique to classify the risk of developing diabetic foot. In this approach, clusters of patients are formed without pre-existing labels. Cluster analysis identifies similarities in the data and responds based on the presence or absence of such similarities in each new sample. In this work, the processed input data vector (representing a patient) was assigned to one of two groups: high risk or low risk of developing diabetic foot. To achieve this, we made use of a competitive neural layer to classify the risk of developing diabetic foot. In this regard, our architecture and objectives were simpler than those systems using MLPs and CNNs.

\section{Methods}

This section describes the captured dataset, the design of the proposed method, and the statistical analysis carried out to identify the variables that most discriminated between highand low-risk patients for developing diabetic foot.

\section{Multivariate dataset}

Our study was developed in an institution for diabetic patients of the Midwest of Minas Gerais, Brazil. The research participants were patients with DM, who were registered in the institution with age greater than or equal to 18 years and who agreed to participate in the research (inclusion criteria), having signed an informed consent form (ICF), complying with the standards of research involving human beings in accordance to Resolution no. 466 of the National Health Council in Brazil, dated December 12, 2012. The anonymity of patient data was ensured through the study methods. The data collection occurred after authorization from the institution and project approval by the Institutional Review Board (IRB) of the University of Campinas (CAAE 66815617.3.00005404).

The sample was randomly chosen and consisted of 250 registered patients from the institution. We approached candidates in the waiting room, while they waited for their 
medical appointment. The interview for data collection was conducted privately in a separate room.

The instrument (questionnaire) used for data collection was adapted in accordance with the scientific parameters of risk factors for the development of diabetic foot proposed by International Consensus on the Diabetic Foot from $2001^{(39)}$ and the DM brochure from the Ministry of Health from $2006^{(1)}$.

The questionnaire was based on self-care habits of patients in relation to their health and foot care, but also included the patient's sociodemographic and socioeconomic information. Each entry in our dataset contained the subject's income, profession, gender, age, years of scholarship, marital status, body mass index (BMI), smoking habit, alcohol use, type of diabetes, presence of hypertension, circulatory problems, burning sensation in the feet, presence of bunion, examination of the feet, wrinkle between the toes, the habit of washing the feet, calluses on the feet, type of footwear (style, material, and construction), time between buying shoes, walking habits (barefoot or not), blood glucose value, if blood glucose monitored, food control, current or previous ringworm on feet, pain in legs and feet, current or previous cracks in the feet, current or previous blister on the feet, shock in feet, warm and reddish feet, removal of cuticles from toes, type of cut of nails on the feet, sock type, action taken when diabetic foot occurred, checking inside the shoe, the position of watching television/reading, length of time standing or walking, type of treatment, time with diabetes, practice of physical activity, tingling in feet, loss of feet sensitivity, numbness in feet, sitting on the feet, edema in feet, change in foot structure, moisturizing of the feet, vision problems, current or previous ingrown nails, use of warm bag on feet, current or previous foot injuries, and presence of leg/foot amputation. In total, each sample (patient input) in our database contained 54 variables. 
The collected data were encoded so that the categorical variables were transformed into floating-point numbers -1.0 or +1.0 , where -1.0 meant that "the value presents lower risk to the diabetic foot condition" and +1.0 , that "the value presents higher risk." To define the conditions with respect to diabetic foot, both the International Consensus on the Diabetic Foot $^{(39)}$ and the brochure about DM from the Ministry of Health ${ }^{(1)}$ were taken into account.

To validate the obtained risk classification, we collected data from a new group of subjects, creating a separate evaluation set. The data of these patients were then analyzed independently by two specialized nurses (with extensive experience in caring for diabetic foot), based solely on patient input (54 variables). During a consensus meeting with a third nurse, the subjects were classified as belonging to the low- or high-risk groups.

\section{Method design}

The design of the proposed method comprised three stages as shown in the block diagram of Figure 1. The first stage corresponded to data normalization, where each variable vector $\left(\mathbf{x}_{j}\right)$ of the dataset was normalized by its maximum absolute value, according to Equation (1).

$$
\mathbf{x}_{\text {jnormalized }}=\frac{\mathbf{x}_{j}}{\max \left(\left|\mathbf{x}_{j}\right|\right)}
$$

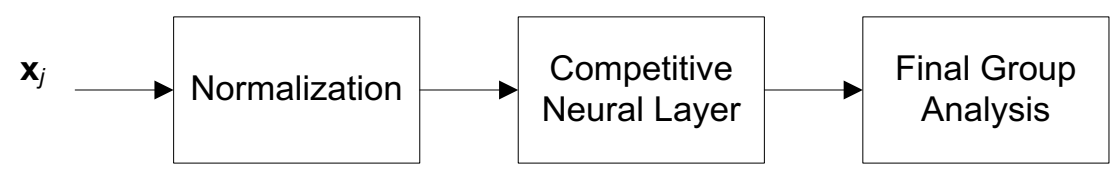

Figure 1. Block diagram of the proposed method design.

In the next stage, a competitive neural layer $(\mathrm{CNL})^{(16)}$ was trained with the input dataset to cluster the data into two groups. The CNL algorithm was unsupervised in nature, which fit well our input data that did not contain labels. The method examined similarities among input samples to enable clustering them. It is a type of competitive learning algorithm 
where the goal is to move each of the representative weight vectors $(\omega j)$ to regions of the vector space that are "dense" in vectors of original data $\mathbf{X}^{(40)}$. The weights were initialized to the centers of the input ranges. The CNL training algorithm updated the weight vectors each time a new input vector $\mathbf{x} \in \mathbf{X}$ was presented to the algorithm. When an input vector was presented to the CNL, all representative weights competed with each other. The winner of this competition was the representative that lay closer (according to some distance measure) to $\mathbf{x}$. Next, the winner was updated so as to move toward $\mathbf{x}$, while the losers either remained unchanged or were updated toward $\mathbf{x}$ but at a much slower rate ${ }^{(40)}$. The training stopped when the maximum number of epochs (repetitions) was reached or when the weights of neurons changed minimally from one epoch to the next.

The CNL architecture is shown in Figure 2. There are two neurons, each one representing a group (or cluster). In this diagram, the input data, containing the patient's information, is represented by variables $x_{1}, x_{2}, \ldots, x_{N}$. The weights of the neural network are represented by $W_{i j}$ and $b_{j}$, so that $i=1,2, \ldots, N$ and $j=1,2$, and $y_{j}$ comprises the outputs. Each neuron performs a simple computation as shown in Equation (2) to give the respective output:

$$
y_{j}=-\left\|\mathbf{x}-\mathbf{W}_{j}\right\|+b_{j}
$$

where the term $-\left\|\mathbf{x}-\mathbf{W}_{j}\right\|$ is the negative Euclidean distance between the input vector $\mathbf{x}=$ $\left[\begin{array}{llll}x_{1} & x_{2} & \ldots & x_{N}\end{array}\right]^{T}$ and the column $j$ of the weight matrix $\mathbf{W}$. 


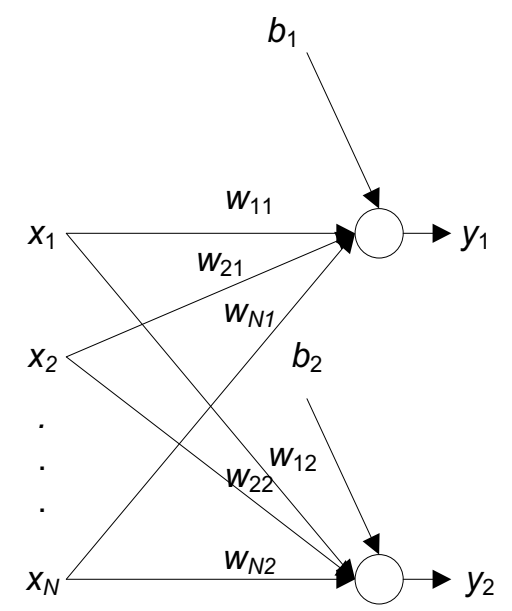

Figure 2. Competitive neural layer architecture.

For every input vector, the competitive neurons "compete" with each other to see which one of them is the most similar to that particular input vector. The winner neuron sets its output $y_{j}=1$, and the other neuron sets its output $y_{m}=0, j \neq m$. To train the CNL, which consists in adjusting the weights of the neural network, the Kohonen Learning Rule ${ }^{(40,41)}$ was used.

The last step of the design of the proposed method (see Figure 1) consisted of defining which of the obtained groups presented the higher risk of developing diabetic foot. It is worth noting that the task of characterizing groups in clustering analysis is not trivial. It depends on the area of application and on the data, as no previous classification is available. Typically, it is carried out via statistical analysis of each group with the aim of finding differences that permit identifying them, with the help of a specialist in the field ${ }^{(40)}$.

\section{Statistical analysis}

In this study, we applied the Student's $t$-test ${ }^{(42)}$ for quantitative variables where a normal distribution was observed. For data with non-normal distributions, we applied the Mann-Whitney $U$ test $^{(42)}$. Finally, to study associations between the qualitative data, we applied the chi-squared test ${ }^{(42)}$. When the assumptions of the chi-squared test were not met, the Fisher's exact test was applied instead ${ }^{(43)}$. For all the analysis, we considered a 
significance level of $5 \%$. This part of the work was carried using the statistical software package SAS (version 9.4).

After statistical analysis, an investigation was carried out by a nurse specialized in diabetic foot diagnosis and prevention. The goal of the specialist nurse was to verify the presence of variables considered to be risk factors for the development of the condition. To do so, the nurse took risk factors defined in the brochure about DM from the Ministry of Health $^{(1)}$ and the International Consensus on the Diabetic Foot ${ }^{(39)}$ into account.

\section{Results}

Out of the total (250 patients), 11 were eliminated due to missing information in the questionnaire. The remaining patients were clustered into two groups by our CNL-based architecture: Group A (GA) with 127 diabetic patients, and Group B (GB) with 112 diabetic patients.

The dataset was predominantly composed of an elderly population $(49.6 \%$ aged 61 or over), with a slight female majority (55.6\%), a monthly income from 120 to 192 US dollars (39.6\%), and a low level of schooling (34.4\% with only 0 to 3 years). It was possible to infer during the data collection process that the overall study population had difficulties understanding information provided by health professionals, the ideal care to be taken with feet, and how to deal with the disease at home ${ }^{(20)}$. Most of the participants were married (52.8\%), had type 2 diabetes (95.2\%), had overweight BMI (36.8\%), and were first positively diagnosed 11 to 20 years before the data collection (34.8\%).

Tables 1 and 2 show the statistical analysis for GA and GB, considering each input variable. Table 1 focuses on quantitative variables. Table 2 refers to qualitative variables (e.g., habits, attitudes, changes on the feet, and sensation). In Table 1, it can be observed that only age presented significant differences between GA and GB, with a $p$-value of 0.0214 . Table 2 presents qualitative variables that had significant difference between GA and GB. Each 
variable is denoted by a short sentence, referring to a patient habit or condition. If the patient had the habit or condition indicated, the answer "yes" was associated with that item. A "no" answer was assigned to patients who never had the habit mentioned in the questionnaire item.

Table 1. Comparison between quantitative variables from Group A and Group B

\begin{tabular}{|c|c|c|c|c|c|c|c|c|c|c|}
\hline Variable & Group & $n$ & Average & $\begin{array}{l}\text { Standard } \\
\text { Deviation }\end{array}$ & Minimum & Q1 & Median & Q3 & Maximum & $p$-value \\
\hline \multirow[t]{2}{*}{ Age (Years) } & $\mathrm{A}$ & 127 & 62.45 & 13.97 & 27.00 & 51.00 & 64.00 & 72.00 & 88.00 & $0.0240 *$ \\
\hline & B & 112 & 58.63 & 11.54 & 32.00 & 50.00 & 59.00 & 67.00 & 84.00 & \\
\hline Years of & A & 127 & 5.50 & 4.50 & 0.00 & 3.00 & 4.00 & 8.00 & 16.00 & $0.6064 * *$ \\
\hline study & B & 112 & 5.56 & 4.05 & 0.00 & 3.00 & 4.00 & 8.00 & 15.00 & \\
\hline Income & A & 127 & 315.21 & 201.77 & 119.69 & 184.33 & 239.38 & 406.95 & 1436.30 & $0.7835^{* *}$ \\
\hline (US dollar) & B & 112 & 306.05 & 201.36 & 119.69 & 191.51 & 239.38 & 359.08 & 1675.68 & \\
\hline Duration of & A & 127 & 12.46 & 8.01 & 1.00 & 5.00 & 10.00 & 17.00 & 43.00 & $0.7959 * *$ \\
\hline Diabetes & B & 112 & 13.13 & 9.04 & 1.00 & 5.50 & 10.00 & 19.00 & 37.00 & \\
\hline \multicolumn{11}{|l|}{ (Years) } \\
\hline BMI & A & 127 & 28.02 & 5.12 & 17.83 & 24.03 & 27.61 & 31.14 & 41.61 & $0.1373 *$ \\
\hline$\left(\mathrm{Kg} / \mathrm{m}^{2}\right)$ & B & 112 & 29.04 & 5.45 & 17.58 & 25.61 & 28.71 & 32.85 & 47.87 & \\
\hline
\end{tabular}

* $P$-value obtained through the unpaired Student's $t$-test.

**P-value obtained by means of the Mann-Whitney U test.

Table 2. Associations between presence of qualitative variables in Groups A and B

\begin{tabular}{llllll}
\hline & \multicolumn{2}{l}{ Group } & & & \\
Variable & A & & B & & P-value \\
& $\boldsymbol{n}$ & $\mathbf{\%}$ & $\boldsymbol{n}$ & $\mathbf{\%}$ & \\
& & & & & \\
\hline Smoking & & & & & $<\mathbf{0 . 0 0 0 1 *}$ \\
No & 119 & 93.70 & 76 & 67.86 & \\
Yes & 8 & 6.30 & 36 & 32.14 & \\
Alcohol use & & & & & $\mathbf{0 . 0 1 9 4 *}$ \\
No & 99 & 77.95 & 72 & 64.29 & \\
Yes & 28 & 22.05 & 40 & 35.71 & \\
Long time standing or walking** & & & & & $\mathbf{0 . 0 3 6 7 ^ { * }}$ \\
No & 52 & 40.94 & 61 & 54.46 & \\
\end{tabular}


Yes

How are your toenails trimmed?

Not close to the nail bed and straight,

without cutting the corners

Close to the nail bed, but in a straight way,

without cutting the corners or close to the

nail bed, following the corners or not close

to the nail bed, but cutting the corners

Removal of cuticles from toes

No

Yes

Current or previous ingrown nails

No

Yes

The habit of washing the feet

No

Yes

Wrinkle between the toes

No

Yes

Calluses on the feet

No

Yes

Use of warm bag on feet

No

Yes

Walking habits (barefoot )

No

Yes

Action taken when any changes occur in

your feet

Use medication or put your feet up or

do nothing, wait for issues to evolve

Go to a health professional

Current or previous foot injuries

No

Yes

Circulation problem
45.54

0.0299*

$\begin{array}{llll}38 & 29.92 & 20 & 17.86\end{array}$

$\begin{array}{llll}89 & 70.08 & 92 & 82.14\end{array}$

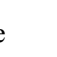

(1)




\begin{tabular}{|c|c|c|c|c|c|}
\hline No & 113 & 88.98 & 86 & 76.79 & \\
\hline Yes & 14 & 11.02 & 26 & 23.21 & \\
\hline Loss of feet sensitivity & & & & & $<0.0001 *$ \\
\hline No & 109 & 85.83 & 38 & 33.93 & \\
\hline Yes & 18 & 14.17 & 74 & 66.07 & \\
\hline Current or previous blister on the feet & & & & & $<0.0001 *$ \\
\hline No & 102 & 80.31 & 48 & 42.86 & \\
\hline Yes & 25 & 19.69 & 64 & 57.14 & \\
\hline Current or previous cracks in the feet & & & & & $<0.0001 *$ \\
\hline No & 82 & 64.57 & 34 & 30.36 & \\
\hline Yes & 45 & 35.43 & 78 & 69.64 & \\
\hline Current or previous ringworm on feet & & & & & $<0.0001$ * \\
\hline No & 69 & 54.33 & 28 & 25.00 & \\
\hline Yes & 58 & 45.67 & 84 & 75.00 & \\
\hline Tingling in feet & & & & & $<0.0001$ * \\
\hline No & 95 & 74.80 & 6 & 5.36 & \\
\hline Yes & 32 & 25.20 & 106 & 94.64 & \\
\hline Numbness in feet & & & & & $<0.0001 *$ \\
\hline No & 95 & 74.80 & 15 & 13.39 & \\
\hline Yes & 32 & 25.20 & 97 & 86.61 & \\
\hline Burning feet & & & & & $<0.0001$ * \\
\hline No & 115 & 90.55 & 46 & 41.07 & \\
\hline Yes & 12 & 9.45 & 66 & 58.93 & \\
\hline Shock on feet & & & & & $<0.0001$ * \\
\hline No & 122 & 96.06 & 54 & 48.21 & \\
\hline Yes & 5 & 3.94 & 58 & 51.79 & \\
\hline Pain in legs and feet & & & & & $<0.0001$ * \\
\hline No & 70 & 55.12 & 33 & 29.46 & \\
\hline Yes & 57 & 44.88 & 79 & 70.54 & \\
\hline Claw toes & & & & & $<0.0001$ * \\
\hline No & 120 & 94.49 & 36 & 32.14 & \\
\hline Yes & 7 & 5.51 & 76 & 67.86 & \\
\hline Change in foot structure & & & & & $<0.0001$ * \\
\hline No & 104 & 81.89 & 59 & 52.68 & \\
\hline Yes & 23 & 18.11 & 53 & 47.32 & \\
\hline Halux valgo & & & & & $0.0392^{*}$ \\
\hline No & 103 & 81.10 & 78 & 69.64 & \\
\hline Yes & 24 & 18.90 & 34 & 30.36 & \\
\hline
\end{tabular}




\begin{tabular}{lccccc} 
Warm reddish feet & & & & & $<\mathbf{0 . 0 0 0 1 *}^{*}$ \\
No & 111 & 87.40 & 55 & 49.11 & \\
Yes & 16 & 12.60 & 57 & 50.89 & \\
Edema in feet & & & & & $<\mathbf{0 . 0 0 0 1 *}^{*}$ \\
No & 79 & 62.20 & 38 & 33.93 & \\
Yes & 48 & 37.80 & 74 & 66.07 & \\
\hline
\end{tabular}

* $P$-value obtained through the chi-square test.

**For the question "Long time standing or walking," the current occupation of the patient was taken into account, i.e., if it required more than four hours a day, it was considered a long time and, therefore, the answer was "yes."

The CNL training performs the data clustering giving more importance to the most discriminative variables and less importance to the least discriminative ones. By analyzing the weights of its neurons, it is possible to find the most discriminative variables. Figure 3 shows the distribution of the weights in each neuron for all input variables. It can be seen that there was a similar behavior of the weight distribution in both neurons. A small variation of the neuron weights from one model to another was found for the majority of variables. Out of 54 variables, 15 variables presented absolute values of weights greater than 0.5 , showing these to be the most discriminating ones. These variables were age (variable 1), type of diabetes (variable 9), BMI (variable 10), food control (variable 12), physical activity (variable 14), smoking (variable 15), presence of hypertension (variable 17) and circulatory problems (variable 18), sensation of shock in the feet and legs (variable 26), presence of bunion (variable 30), visual changes (variable 33), the habit of washing the feet (variable 39), presence of calluses in the feet (variable 42), presence of a wound (variable 53) and/or amputation (variable 54). 


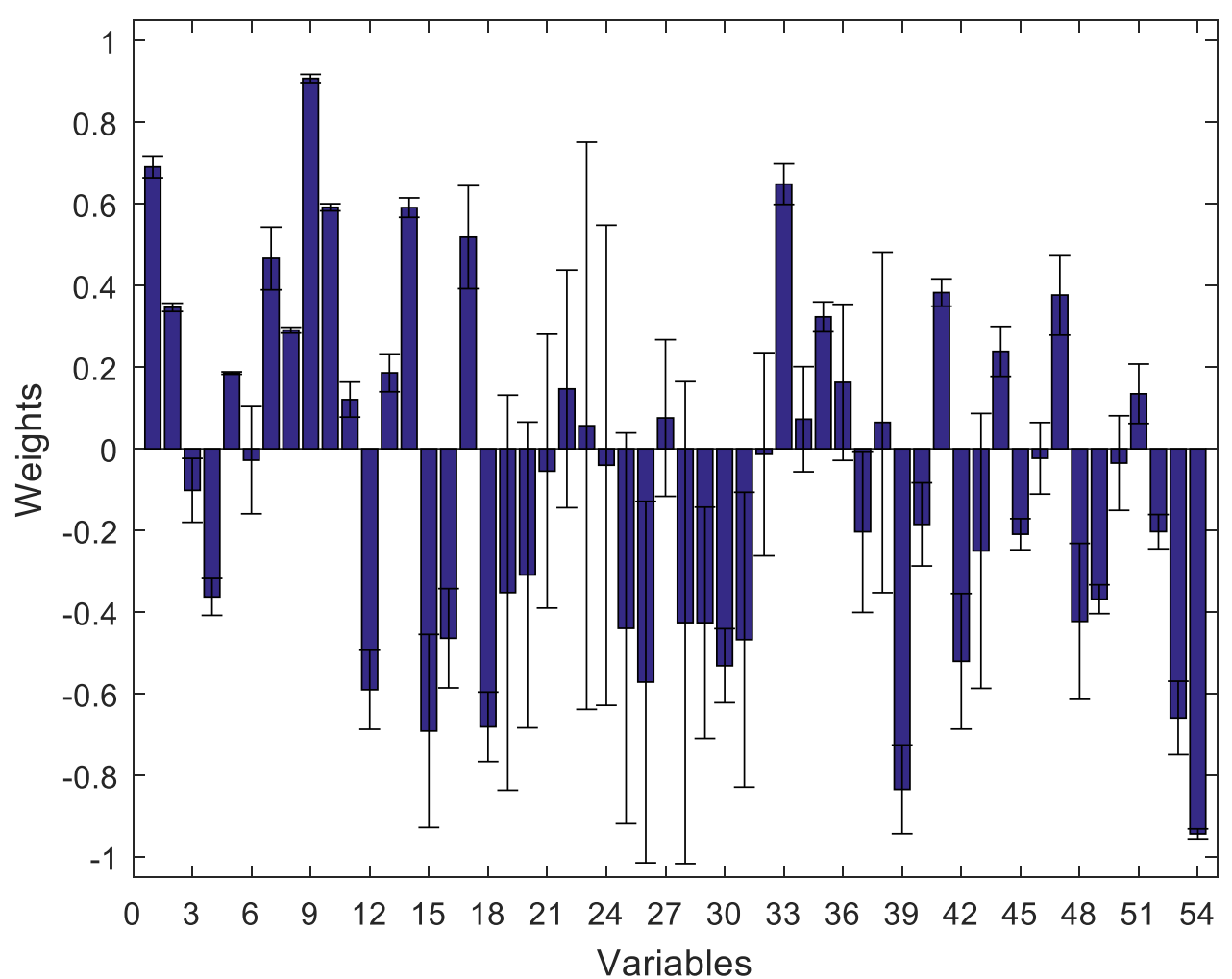

(a)

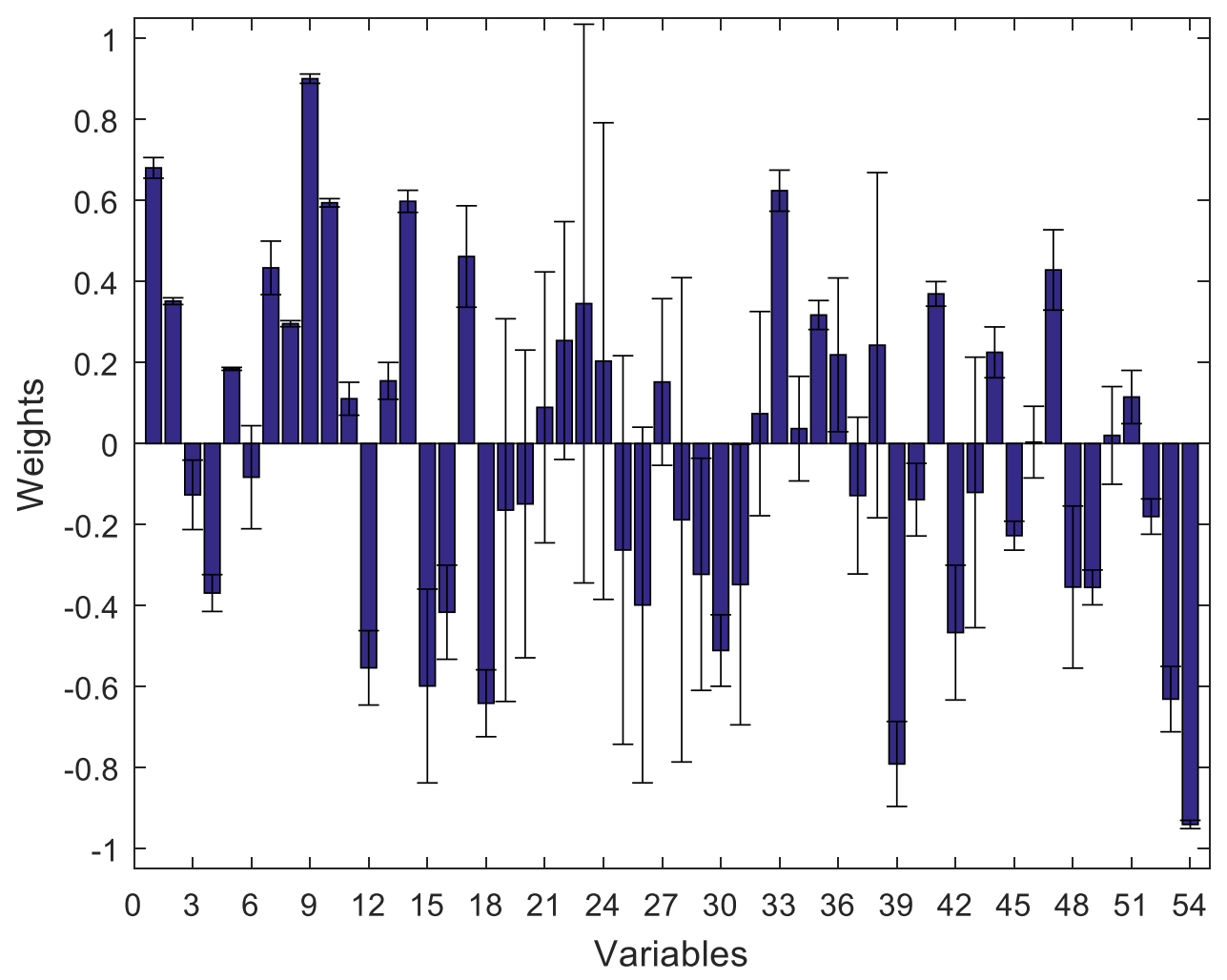

(b) 
Figure 3. Weights (mean \pm standard deviation for the 20 models) of the neurons of the proposed CNL in terms of the variables: (a) neuron that represents Group A; (b) neuron that represents Group B.

Our model was applied to the evaluation set composed of data from 73 subjects. It found 15 true positives, 9 false positives, 49 true negatives, and no false negatives, reaching a sensitivity of $71 \%$, a specificity of $100 \%$, and an accuracy of $90 \%$.

\section{Discussion}

The factors that primarily described differences between the two groups after statistical analysis were the following: smoking; loss of sensation in the feet; having or had blisters, cracks, ringworm in feet; feeling tingling, numbness, burning, shock, and pain in the feet; experiencing hot, red, or swollen feet; having or had have ingrown toenails and calluses on the feet; and applying a hot water bottle to the feet. It is important to note that most participants who had these characteristics belonged to Group B. Based on this analysis, we concluded that Group B represented the diabetic people who have the greatest risk to develop diabetic foot. These factors, combined with a positive DM diagnosis, may indicate the presence of sensory alterations which are predisposing conditions to the appearance of wounds ${ }^{(39)}$.

After training, our proposed clustering algorithm showed that the characteristics considered most relevant for the classification in the risk groups were age, type of diabetes, BMI, food control, physical activity, smoking, presence of hypertension and circulatory problems, sensation of shock in the feet and legs, presence of bunion, visual changes, the habit of washing the feet, presence of calluses in the feet, and presence of a wound and/or amputation (see Figure 3). 
Eight of the 14 characteristics identified by the proposed system were also identified via statistical analysis: age, smoking, change in circulation, sensation of shock, presence of bunion, the habit of washing the feet, presence of calluses, and wounds on the feet.

Advanced age, when associated with other factors such as the low education level, is considered to be a risk factor for diabetic foot development ${ }^{(44,45)}$, in spite of this, in our study, Group A (and not Group B, as expected) had older participants. It is known that smoking is one of the modifiable factors that aggravate the clinical condition of patients with diabetes. Due to neurological alterations caused by diabetes, loss of sensitivity in the limbs should be considered as a possible risk factor for the development of lesions. Habits such as improper nail cutting, with the possibility of ingrown nails, removal of cuticles, with the possibility of infection in this area, and appearance of callus, are also factors that facilitate the appearance of diabetic foot ${ }^{(39,46)}$.

Diabetic neuropathy is conceptualized as the presence of symptoms and/or signs of peripheral nerve dysfunction in patients with diabetes, after excluding other causes. It may also be classified according to clinical manifestations. Diabetic foot injuries usually result from a combination of two or more risk factors occurring concomitantly, such as loss of sensation and the presence of blisters on the feet. Sensory neuropathy is associated with loss of pain sensitivity, lack of perception of pressure, and presence of extreme temperatures. Due to the loss of these modalities, the stimuli to feel some injury or trauma are diminished or even imperceptible, and may result in ulceration ${ }^{(46-47)}$.

Group A revealed a greater number of people who did not have any changes in blood circulation. The vascular disease that affects the lower limbs is the most common form of peripheral vascular disease. This disease can lead to intermittent claudication or pain at rest, even causing ulceration and gangrene. Estimates have shown that the incidence of peripheral 
and cerebrovascular vascular disease is two to four times higher in diabetic people than in the general population $^{(10,48)}$.

In addition, the great majority of Group A had no difference in the structure of the feet, whereas most of those in Group B had a difference in the skeletal structures of the feet. Sensory motor neuropathy can lead to muscle atrophy of the foot muscles, generating imbalance in the flexor and extensor muscles. This can lead to deformities such as "claw toes," "hammer toes," overlapping toes, prominences of the metatarsal heads, and bunion. These deformities lead to different pressure points in the plantar region, which overload certain areas causing calluses and can progress to ulceration after intense walking (perforating plantar ulcers $)^{(14,49)}$.

The great proportion of risk factors determined in Group B revealed the importance of an immediate intervention with those people by a specialized nurse with the aim of preventing and treating risk factors of diabetic foot. The nurse had to organize an agenda of individualized and systematized consultations for each of the participants, in order to create the means that help them in the process of therapeutics and prevention. For members of the group with a lower frequency of risk factors (Group A), it was possible to create health education groups focused on prevention of diabetic foot.

In addition, the system enabled the accessing and updating of the most relevant information for the systematic control, that is, the modifiable risk factors of each patient at each nursing consultation. The inspection of the feet must be made so that the patient will carefully check each part of the foot, verifying the interdigital spaces, sole, calcaneus, toes, and the top of the feet. Obese individuals, at an advanced age and/or with low visual acuity, may find difficulties in performing the inspection. In these cases, it is essential that the partner and/or family members are instructed as to the importance of this care ${ }^{(46-49)}$. Family and social support are important, especially for those with sight loss and walking difficulties ${ }^{(44-45)}$. 
Because of the nature of DM, individuals suffering from it who live alone, without friends or relatives, social or religious activities, and access to education, and are of a low socioeconomic status, are considered individuals who are more likely to develop diabetic foot and undergo amputation ${ }^{(44-45)}$.

Notably, the increasing number of diabetic patients has made it more difficult for health professionals to optimize care; consequently, most clinical exams and interventions necessary to prevent complications may not be correctly performed. Therefore, a triage is very useful, as it would detect those who need immediate intervention. Accordingly, a healthcare professional can organize care by the level of priority. Although there is no scientific parameter that indicates the risk for the development of diabetic foot without performing clinical exams, our system showed good performance, since the statistical analysis indicated two different groups in the dataset. In addition, the obtained results agreed with the classification performed by specialists (90\% of accuracy, $100 \%$ of specificity, and $71 \%$ of sensitivity). It is worth to noting that the classification by specialists was a difficult task ${ }^{(23)}$, since no clinical exam was performed, and there was a great number of risk factors available to be investigated. In addition, high specificity is important in the screening processes, which was the case of our study. Patients identified as having a low risk of developing diabetic foot were not strictly monitored after this phase.

With the risk classification given by the proposed method, the healthcare team may focus their work on people who are at risk of foot complications, avoiding the diabetic foot complications more rapidly and effectively.

Regarding the computational complexity of the proposed neural network, it requires only the computation of two Euclidean distances between the vector of input variables and the weight vectors, which is very simple for implementation purposes. By using a notebook with an Intel(R) Core(TM) i7-6500U CPU @ 2.50GHz processor, $2601 \mathrm{MHz}, 16 \mathrm{~GB}$ of RAM, it 
took about $0.32 \mathrm{~s}$ to process the input variables of a patient via MatLab software. Therefore, the proposed method may be implemented in simple computers using the software that was developed.

Compared with related work mentioned in the literature review section, we may conclude that the proposed method differs from those in the following respects: (i) it is noninvasive; (ii) it does not require any image processing, (iii) it does not require a sensor to monitor the patient's feet, and, primarily, (iv) it deals with the prediction of the risk of developing diabetic foot.

\section{Limitations of the Work}

It is worth mentioning that the proposed method is based on subjective information provided by participants with DM, which may lower the quality of the risk prediction. It is known that reliability of patient-reported data is low or unpredictable ${ }^{(50)}$, as well as in the case of highly motivated subgroups of people with DM. As previously mentioned before, the interview for data collection of this work was conducted privately in a separate room by a health specialist. In this case, the patient might have felt embarrassed for not carrying out foot care habits and, therefore, could have answered the questions in an unreliable way. We believe that the accuracy of the proposed method may be improved if methods to ensure the data quality are applied before designing the risk classifier.

Another limitation of this work is that we did not demonstrate that those patients who were classified as being at a high risk did develop diabetic foot, whereas those classified as low risk did not. Such a validation would demand a larger study ${ }^{(23)}$. On the other hand, if the patient is classified as high risk, our tool would allow immediate intervention toward improving self-care to avoid the development DFU.

\section{Final Remarks}


We proposed an unsupervised method to classify patients with DM who have high risk of developing diabetic foot with an accuracy of $90 \%$. The proposed system takes advantage of using information collected through a questionnaire and without clinical exams. The variables that most discriminate low- from high-risk patients are in accordance with the findings from other studies. Thus, the method's use may optimize nursing work by quickly screening people with DM that require priority follow-up in the prevention of diabetic foot. Our system cannot replace the examination of the patient, but it could be a valuable asset assisting nurses to face the enormous burden of diabetes.

The contributions of our work are threefold: i) We collected a multivariate dataset for diabetic foot screening from non-invasive information, ii) we developed an unsupervised method for screening patients who are likely to develop diabetic foot based on multivariate data and, finally, iii) we analyzed the contributions of variables to the patient screening process and their relationship to findings from our clustering solution.

The authors intend to collect and use further risk classification annotations by experts to build supervised machine learning solutions for diabetic foot risk screening in the near future. We intend to develop an application that implements better quality control in the future, limiting uncertainty factors, and making collected information more reliable.

With this work, we expect to improve the accuracy of diabetic foot risk classification by providing a simple means to stratify the risk of developing DFUs and improving the care of patients who need it most.

\section{Acknowledgments}

The authors would like to thank the Brazilian organs: Conselho Nacional de Desenvolvimento Científico e Tecnológico - National Council for Scientific and Technological Development (CNPq) and Coordenação de Aperfeiçoamento de Pessoal de 
Nível Superior - Coordination for the Improvement of Higher Education Personnel (CAPES) for their support of this work.

\section{References}

1. Brasil. Ministério da Saúde. Diabetes Mellitus. Cadernos de Atenção Básica n. ${ }^{\circ}$ 16, Série A. Normas e Manuais Técnicos. Brasília, DF; 2006. (Portuguese only). Disponível em: http://bvsms.saude.gov.br/bvs/publicacoes/diabetes_mellitus_cab16.pdf. (Portuguese only)

2. El-Gayar O, Timsina P, Nawar N, Eid W. A systematic review of IT for diabetes selfmanagement: Are we there yet? Int J Med Inform. 2013;82(8):637-52. doi: 10.1016/j.ijmedinf.2013.05.006. Epub 2013 Jun 21.

3. International Diabetes Federation. IDF Diabetes Atlas, nona edição, 2019.

4. International Diabetes Federation. Diabetes Atlas, 8th ed [Internet]. Brussels: Belgium.; 2017. Available from: http://www.diabetesatlas.org/.

5. Brasil. Ministério da Saúde. Vigitel Brasil 2016: vigilância de fatores de risco e proteção para doenças crônicas por inquérito telefônico [Internet]. Secretaria de Vigilância em Saúde. Departamento de Vigilância de Doenças e Agravos não Transmissíveis e Promoção da Saúde, editor. Brasília: Ministério da Saúde; 2017. p. 160. Available from:http://portalarquivos2.saude.gov.br/images/pdf/2018/marco/02/vigitel-brasil-2016.pdf.

6. Bakker K, Apelqvist J, Schaper NC. And on behalf of the International Working Group on the Diabetic Foot Editorial Board: 'Practical guidelines on the management and prevention of diabetic foot 2011. Diabetes Metab. Res. Rev. 2012;28(Suppl 1):225-31. doi: 10.1002/dmrr.2253.

7. Mor P, Aviv S, Dongwen W, Eddy K. Using multi-perspective methodologies to study users' interactions with the prototype front end of a guideline-based decision support system for diabetic foot care. International Journal of Medical Informatics. 2009;78(7):482-93. doi: 10.1016/j.ijmedinf.2009.02.008. 
8. Raspovic KM, Wukich DK. Self-reported quality of life and diabetic foot infections. J. Foot Ankle Surg. 2014;53(6):716-19. doi: 10.1053/j.jfas.2014.06.011. Epub 2014 Aug 13.

9. Adam M, Ng EYK, Tan JH, Heng ML, Tong JWK, RajendraAcharya U. Computer aided diagnosis of diabetic foot using infrared thermography: A review. Computers in Biology and Medicine. v 91, 326-336, 2017.

10. Leese GP, Stang D, Pearson DW. A national approach to diabetes foot risk stratification and foot care. Scott. Med. J. 2011;56(3):151-55. doi: 10.1258/smj.2011.011113.

11. Shearman CP, Pal N. Foot complications in patients with diabetes. Surg. Oxf. 2013;31(6): 240-45. doi: 10.1016/j.mpsur.2010.02.002.

12. Chellan G, Srikumar S, Varma AK, ET AL. Foot care practice - the key to prevent diabetic foot ulcers in India. Foot. 2012;22(4):298-302. doi: 10.1016/j.foot.2012.08.007.

13. Brasil. Ministério da Saúde. Manual do Pé diabético: Estratégia para o cuidado da pessoa com doença crônica. Brasília, DF; 2016. (Portuguese only). Disponível em: http://www.as.saude.ms.gov.br/wp-content/uploads/2016/06/manual_do_pe_diabetico.pdf. (Portuguese only)

14. Monteiro-Soares M, Boyko EJ, Ribeiro J, Ribeiro I, Dinis-Ribeiro M. Risk stratification systems for diabetic foot ulcers: a systematic review. Diabetologia. 2011;54(5):1190-99.doi: 10.1007/s00125-010-2030-3.

15. Boyko DJ, Ahroni JH, Cohen V, Nelson KM, Heagerty PJ. 'Prediction of diabetic foot ulcer occurrence using commonly available clinical information: the Seattle diabetic foot study’. Diabetes Care 2006, 29(6):1202-07.doi:10.2337/dc05-2031.

16. Haykin, S. Neural networks and Learning Machines, 3 ed., Prentice Hall; 2008.

17.Richard JL, Reilhes L, Buvry S, Goletto M, Faillie JL. Screening patients at risk for diabetic foot ulceration: a comparison between measurement of vibration perception threshold 
and 10-g monofilament test. Arch Intern Med. 1998;158(2):157-162. doi:10.1001/archinte.158.2.157.

18. Lei N 7.498/86, de 25 de junho de 1986. COFEN Conselho Federal de Enfermagem. Acesso em: 08-09-17. (Portuguese only)

19. Wagner FW. The disvascular foot: a system for diagnosis and treatment. Foot and Ankle. 1981;2(2):64-122.

20. Armstrong DG, Lavery LA, Harkless LB. Validation of a diabetic wound classification system: the contribution of depth, infection and vascular disease to the risk of amputation. Diabetes Care $199821855-859$

21. Leese GP, Reed F, Green V, et al. Stratification of foot ulcer risk in patients with diabetes: a population based study. Int. J. Clin. Pract. 2006;60(5):541-45.doi: 10.1111/j.13685031.2006.00899.x.

22. Scottish Intercollegiate Guidelines Network. 116 Management of Diabetes: A National Clinical Guideline. Edinburgh: SIGN 2010. Disponível em: www.sign.ac.uk/pdf/sign116.pdf (accessed 10 September 2017).

23. Monteiro-Soares M, Boyko EJ, Ribeiro J, Ribeiro I, Dinis-Ribeiro M. Risk stratification systems for diabetic foot ulcers: a systematic review. Diabetologia 2011;54(5):1190-99.doi: $10.1007 / \mathrm{s} 00125-010-2030-3$

24. Silva RN, Ferreira ACBH, Ferreira DD, Barbosa BHG. Non-invasive method to analyse the risk of developing diabetic foot. Healthcare Technology Letters. 2014;1:109-13. doi: 10.1053/j.jfas.2014.06.011.

25. Farzi S, Kianian S, Rastkhadive I. Predicting serious diabetic complications using hidden pattern detection. International Conference on Knowledge-Based Engineering and Innovation (KBEI), $4^{\text {th }}$, IEEE. 2017. Iran University of Science and Thecnology. 
26. Heald A, Lunt M, Rutter MK, et al. Developing a foot ulcer risk model: what is needed to do this in a real-world primary care setting? Diabetic Medicine. 2019; 36, 1412-1416.

27. Crawford F, Cezard G, Chappell FM. Systematic Review or Meta-analysis The development and validation of a multivariable prognostic model to predict foot ulceration in diabetes using a systematic review and individual patient data meta-analyses. Diabetic Medicine. 2018, 35, 1480-1493.

28. Goyal M, Reeves ND, Davison AK, Rajbhandari S, Spragg J, Yap MH. DFUNet: Convolutional Neural Networks for Diabetic Foot Ulcer Classification. IEEE Transactions on Emerging Topics in Computational Intelligence, 2018, 2471-285X:1-12. Doi: 10.1109/TETCI.2018.2866254

29. Singh K, Singh VK, Agrawal NK, Gupta SK, Singh K. Association of Toll-Like Receptor 4 Polymorphisms with Diabetic Foot Ulcers and Application of Artificial Neural Network in DFU Risk Assessment in Type 2 Diabetes Patients. BioMed Research International, 2013. http://dx.doi.org/10.1155/2013/318686.

30. Goyal M, Reeves ND, Davison AK, Rajbhandari S, Yap MH. "Robust Methods for Real-Time Diabetic Foot Ulcer Detection and Localization on Mobile Devices", Biomedical and Health Informatics IEEE Journal of. 2019, 23(4): 1730-1741.

31. Ohura N, Mitsuno R, Sakisaka M, Terabe Y, Morishige Y, Uchiyama A, et al. Convolutional neural networks for wound detection: the role of artificial intelligence in wound care. Journal of Wound Care. 2019, 28(10). https://doi.org/10.12968/jowc.2019.28.Sup10.S13

32. Gomes AA, Suda EY, Sartor CD, Ortega NRS, Watari R, Vigneron V, Sacco ICN. Use of artificial intelligence methods for classifying diabetic patients with polyneuropathy. Diabetol Metab Syndr. 2015; 11;7(Suppl 1). doi: 10.1186/1758-5996-7-S1-A4. PMCID: PMC4653495 
33. Li Z, Wang D, Dey N, Ashour AS, Sherratt RS, Shi F. Plantar pressure image fusion for comfort fusion in diabetes mellitus using an improved fuzzy hidden Markov model. Biocybernetics and Biomedical Engineering, 2019, V39, (3):742-752. https://doi.org/10.1016/j.bbe.2019.06.007

34. Wang L, Pedersen PC, Agu E, Strong DM, Tulu B. Area Determination of Diabetic Foot Ulcer Images Using a Cascaded Two-Stage SVM-Based Classification. IEEE Trans Biomed Eng. 2017 Sep;64(9):2098-2109. doi: 10.1109/TBME.2016.2632522

35. Cruz-Vega I, Peregrina-Barreto H, Rangel-Magdaleno JJ, Ramirez-Cortes JM. A comparison of intelligent classifiers of thermal patterns in diabetic foot. 2019. IEEE International Instrumentation and Measurement Technology Conference (I2MTC).

36. Priyadharshini R, Saraswathy Gr, Gopalakrishna G, Bhabendra N, Viswanathan V. Standardization of Foot Sizes of Patients with Diabetic Foot Ulcer through Anthropometric Survey. 2017, Journal The Anthropologist, v28 (3). DOI:10.1080/09720073.2017.1335718

37. Rabbani KS, Ishraque SMZ, Islam MS, Rabbani RM. Improvisation of an Optical Pressure Sensor Based Dynamic Foot Pressure Measurement System. 2013, Bangladesh Journal of Medical Physics, 4(1), 51-58. https://doi.org/10.3329/bjmp.v4i1.14687

38. Hernandez-Contreras D, Peregrina-Barreto H, Rangel-Magdaleno J, Gonzalez-Bernal J. Narrative review: Diabetic foot and infrared thermography Infrared. Physics \& Technology. 2016; 78: 105-117. https://doi.org/10.1016/j.infrared.2016.07.013.

39. International Working Group on the Diabetic Foot. International Consensus on the Diabetic Foot. M Wijnandts, Department of Internal Medicine, Academic Hospital Maastricht, Netherlands, f13, 2001.

40. Theodoridis S, Koutroumbas K. Pattern Recognition. 4th edition, 984p, 2008.

41. Kohonen, T., Self-Organizing and Associative Memory, New York, Springer-Verlag, 1984. 
42. Pagano M, Gauvreau K. Principles of Biostatistics, Ed. Thomson, São Paulo, 2004.

43. Mehta CR, Patel NRA. Network algorithm for performing Fisher's exact test in rxc contingency tables. JASA, 1983; 78(382):427-434.

44. Armstrong DG, Lavery LA. Clinical Care of the diabetic foot. American Diabetes Association, 3 edition, 2016.

45. Gomides DS, Villas-Boas LCG, Coelho ACM, Pace AE. Self-care of people with diabetes mellitus who have lower limb complications. Acta Paulista de Enfermagem, 2013; 26(3):28993.

46. International Best Practice Guidelines: Wound Management in Diabetic Foot Ulcers. Wounds International, 2013. Available from: www. woundsinternational.com

47. Nemcová J, Hlinková E. The efficacy of diabetic foot care education. Journal of Clinical Nursing, 2013, 23: 877-82. doi: 10.1111/jocn.12290.

48. Chunlan M, Jim W, Patrick P, Jan S. Empowering patients with essential information and communication support in the context of diabetes. International Journal of Medical Informatics, 75, (8):577 - 96; 2006.

49. Diretrizes da Sociedade Brasileira de Diabetes: 2014-2015/Sociedade Brasileira de Diabetes; [organização José Egidio Paulo de Oliveira, Sérgio Vencio]. - São Paulo: AC Farmacêutica; 2015. (Portuguese only)

50. Ladyzynski P, et al.: Reliability of blood glucose self-monitoring and its influence on glycemic control in highly motivated type 1 diabetic patients. Diabetes Care 1999 May; 22(5): 854-856, doi: $10.2337 /$ diacare.22.5.854a) 